\title{
Desarrollo de un nuevo sistema de gestión de la investigación universitaria
}

\author{
Rosa Fàbregas*, Andreu Grau*, Sònia Ruiz*
}

Resumen: Se explica el desarrollo de un programa informático para la gestión de los recursos de investigación. Si bien dichos recursos son clave en la consecución de resultados científicos la literatura sobre el campo es aún escasa. Es por ello que el enfoque que propone TAIR es novedoso pues parte del trabajo diario de los gestores de los recursos de investigación y basa su metodología en las necesidades del personal técnico y administrativo que desarrolla esta tarea. En concreto se vislumbra la gestión de los recursos de investigación como un proceso global en el que la identificación de las convocatorias es el elemento clave. A partir de esta identificación se llega hasta la concesión de ayudas, su divulgación en forma de trabajo científico y su posterior inclusión en el curriculum vitae del investigador. En conclusión, se propone que la gestión de los recursos de la investigación sea un proceso completo e integrado con la producción científica mediante el programa informático TAIR.

Palabras Clave: Gestión de los recursos de investigación, TAIR, programarlo.

\section{Development of a new system for university research management}

Abstract: The development of a software program for the management of research resources is explained. Although this kind of resource is of utmost importance for obtaining scientific results, references related to this topic are sparse. The approach proposed by TAIR is novel due to its being based on the daily work of research resources managers and its methodology, on the requirements of technical and administrative staff. Specifically, one could say that the management of research resources is a global process which is set in motion when a public call has been identified. It then proceeds with the grant being awarded, the results published in a scholarly paper, and the citation added to the researchers' CVS. In conclusion, this article recommends that the management of research resources should be a full process integrated into academic production by means of the TAIR software.

Keywords: Management of research resources, TAIR, software.

\section{Introducción}

La producción científica de las universidades y concretamente los artículos de difusión internacional son, a día de hoy, el valor con el que se mide la calidad

* Universitat Autònoma de Barcelona. Correo-e: Rosa.Fabregas@uab.cat; Andreu.Grau@uab.cat; Sonia.Ruiz@uab.cat.

Recibido:06-04-2011;2. ${ }^{a}$ versión: 16-02-2012; aceptado: 17-05-2012. 
de estas instituciones en lo que a capacidad investigadora se refiere (IribarrenMaestro y Sanz, 2008; Moros y Bondons, 2003; Argimbau, 2006). Es por ello que el principal objeto de estudio de bibliómetras y documentalistas dentro del campo de la investigación universitaria atañe habitualmente a la recolección de datos de esta índole y su posterior incorporación a los sistemas de gestión de la información de las universidades. No obstante, hay una parte previa a la publicación del documento con los resultados científicos que habitualmente es obviada por los técnicos de la información. Esta parte es la que hace referencia a la obtención de fondos para llevar a cabo dicha producción, así como la gestión de los mismos durante su ciclo de vida. Este proceso, sin el cual la obtención de conocimientos científicos seria, si bien no imposible, sí mucho más complicada, se conoce como Gestión de los Recursos de Investigación (Baras, C., 2004).

Dicha gestión también necesita, igual que la gestión de la producción científica, de un conjunto de métodos e instrumentos que la hagan eficaz. Y no sólo como medio de asegurar una correcta tramitación de las ayudas. Los datos de gestión de los recursos solicitados y obtenidos van a ser necesarios en el proceso de análisis estratégico y toma de decisiones por parte de los responsables de la universidad (Alvarez, 2009).

Aún así, a día de hoy y en consonancia con el menor interés que suscita la gestión frente a la producción científica, el estudio de estos procesos y sus instrumentos afines son más bien escasos en nuestra geografía. Las universidades gestionan especialmente la producción científica y el currículum de los investigadores con herramientas ad-hoc, dejando para las gestiones económicas de las universidades la parte del proceso que financia la investigación. Existen asimismo herramientas para gestionar los proyectos o grupos de investigación, el personal, los recursos obtenidos, etc. Pero no existen herramientas para gestionar integralmente las ayudas concedidas y gestionadas por una universidad, desde que se convocan hasta que fructifican en una publicación. Esto es, a nuestro parecer, un error, puesto que la gestión de las ayudas y la producción científica son procesos que están ligados y que deben tratarse de forma integral.

En este artículo se reseñará el proceso de desarrollo de un software informático para la Gestión de los recursos de la Investigación mediante un caso concreto, el de la Universitat Autònoma de Barcelona, destacando la importancia que tiene dentro de un sistema científico el correcto diseño de un programario de este tipo.

\section{2. ¿Gestión de los recursos de la investigación?}

Definimos la Gestión de los Recursos de la Investigación como el conjunto de procesos administrativos, técnicos y de asesoría que permiten gestionar de manera operativa los fondos y recursos necesarios para llevar a cabo una actividad científica. Cualquier persona que haya tenido contacto con el mundo académico seguramente ha debido recurrir a una o más actividades encaminadas a gestionar su carrera investigadora; una ayuda para ir a un congreso, una beca, 
un proyecto de investigación, etc. Todo ello forma parte de los procesos habituales de trabajo en las universidades y más concretamente en sus servicios de gestión de recursos de la investigación.

Este conjunto de actividades, como toda gestión, necesita de unos circuitos y un programa adecuado para su correcto cumplimiento (Argimbau, 2004). Es por ello que un software creado a tal efecto va a ser una pieza fundamental del sistema. Pero el dato más relevante para comprender la importancia de un buen sistema de gestión de la investigación es el crecimiento exponencial de los recursos que las administraciones dedican a la investigación científica.

Si vemos la figura 1 podemos comprobar que desde mediados de los años ochenta hasta nuestros días el número de proyectos de investigación gestionados por la UAB se ha multiplicado por quince.

FIGURA 1

Ayudas a la investigación tramitadas por la UAB (sin becas)

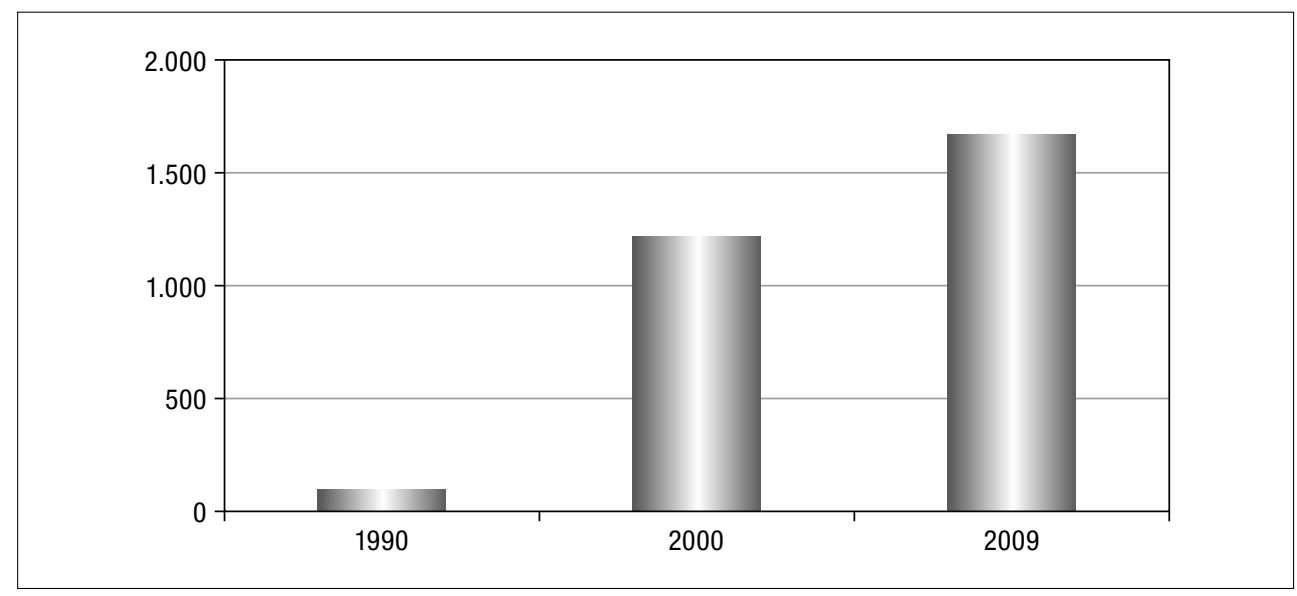

En paralelo a este aumento, en la financiación también han evolucionado los sistemas informáticos que la gestionan. Ahora bien, su desarrollo ha sido a menudo el fruto de la improvisación y no de una estrategia planificada. El software TAIR (Tramesa d'Ajudes a la Recerca) es el resultado de aplicar la experiencia de trabajo del Area de Gestió de la Recerca de la UAB a un proyecto centrado sólo en los procesos propios de este ámbito.

\section{Antecedentes}

El antecedente directo de TAIR fue la aplicación informática Fénix, desarrollada conjuntamente por la Universitat Autònoma de Barcelona, la Universitat Pompeu Fabra y la Universitat Politècnica de Catalunya. Fénix era una aplicación 
cliente-servidor y entre sus principales características constaba la de contener en un mismo sistema un módulo para la Gestión de los Recursos de la Investigación y otro para la Gestión de la Producción Científica. De tal manera que una solicitud de una convocatoria podía dar como resultado una actividad dentro del curriculum del investigador.

\section{TAIR}

Esta visión del negocio ha continuado siendo la médula espinal del sistema en TAIR, si bien en una versión más depurada. El flujo de trabajo de TAIR es el que se muestra en la figura 2.

\section{FIGURA 2}

Flujo de trabajo en la gestión de recursos de investigación

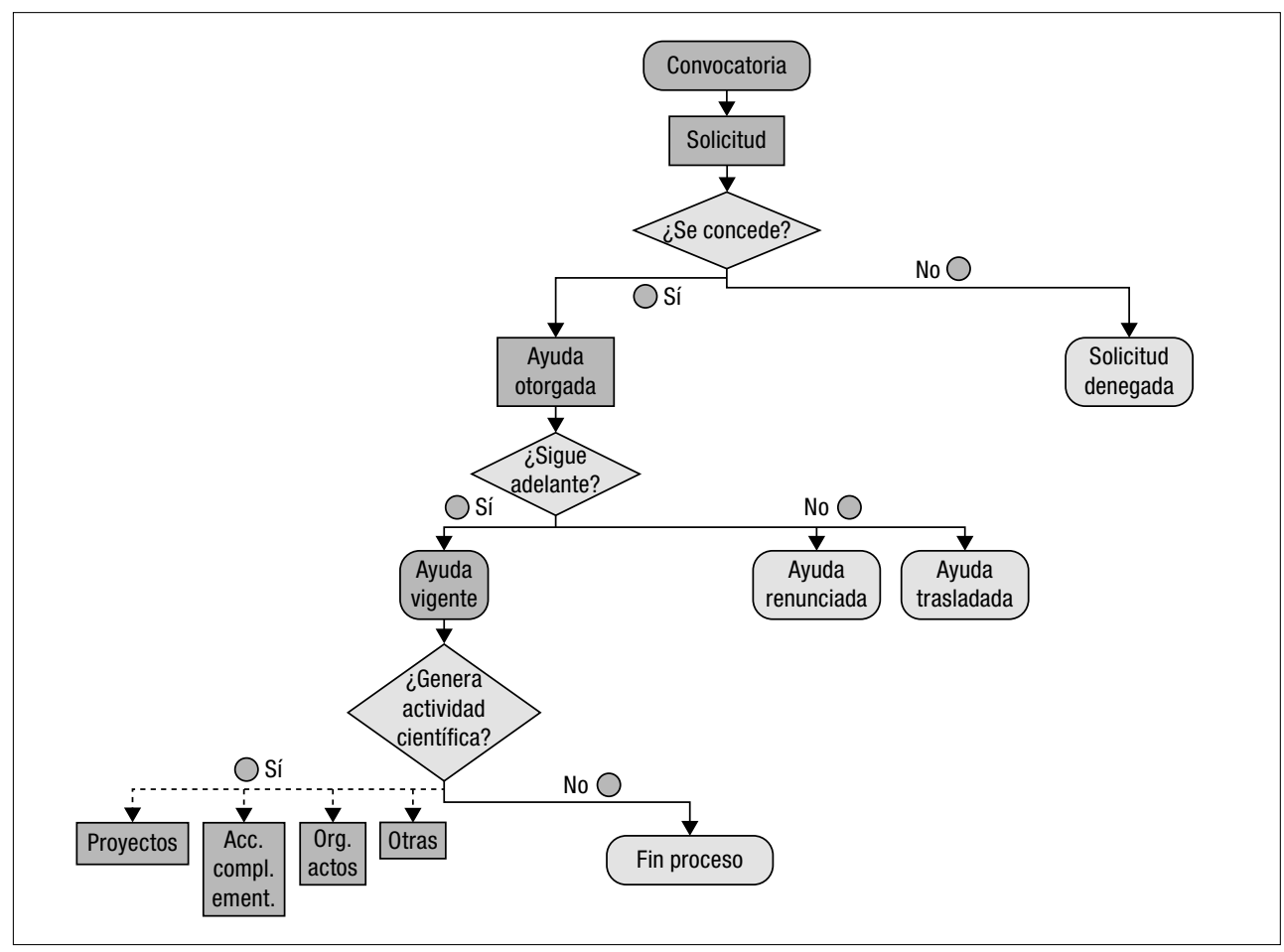

Como vemos se parte de una idea integral de la Gestión de la Investigación, en la que una convocatoria acaba dando lugar a una actividad científica. Esta idea es a nuestro entender novedosa, puesto que la aplicación se desarrolla en función de la gestión real que documentalistas y administradores tienen de la 
materia y no al revés. A menudo, vemos como son los usuarios quienes tienen que amoldarse a las decisiones de técnicos informáticos cuando en la mayoría de los casos debiera ser el usuario, que es quien conoce el trabajo, quien dictaminase los procesos.

La convocatoria es la clave que da sentido a este modelo. Es en la convocatoria donde se va a clasificar la tipología de ayuda. La correcta clasificación es, como en todo sistema de gestión de la información, un proceso básico para el posterior tratamiento de los datos. A partir de esta clasificación (Beca, Estancia, Congreso...) las solicitudes se tramitarán en la aplicación de la manera más adecuada a su tipología. Piénsese que ello puede tener diversas repercusiones en la gestión de la ayuda. Desde repercusiones económicas, hasta en la composición del equipo investigador, etc. Asimismo, la posibilidad de llevar a cabo un buen análisis de la información también será posible si ésta se ha clasificado correctamente.

A partir de la convocatoria se informan en TAIR todas las solicitudes que presentan los investigadores de la universidad. En las solicitudes se introduce una información mínima, puesto que parte de ellas van a ser denegadas y se evita así cargar innecesariamente de trabajo al responsable de su introducción. En aquellas solicitudes que se resuelven favorablemente la información se traspasa, automáticamente, a un mantenimiento de ayudas concedidas, sin necesidad de volver a repicar los datos básicos. Todas las solicitudes deben ir ligadas a una convocatoria. Debe evitarse tener solicitudes huérfanas, es decir, sin estar referenciadas a una convocatoria.

Finalmente, el mismo proceso automático se da en el traspaso de datos de las ayudas a las actividades curriculares. Una ayuda para estancias de investigación en el extranjero, por ejemplo, repercute en una actividad curricular de estancias científicas de investigación, sin necesidad de introducir los datos en una nueva aplicación. Esta actividad curricular pasa a formar parte del curriculum del investigador. Este curriculum, como se verá, puede ser extraído por el investigador con un módulo informático ligado a TAIR. Se da pues, en TAIR, un tratamiento completo de todo el ciclo de ayudas a la investigación.

TABLA I

Convocatorias gestionadas actualmente por TAIR

\begin{tabular}{l|l}
\hline Proyectos & $\begin{array}{l}\text { Proyectos del Plan Nacional, Generalitat, FIS, fundaciones pri- } \\
\text { vadas. }\end{array}$ \\
\hline Becas & Becas MEC, MICINN, Generalitat, propias universidad. \\
\hline Incorporación de personal & Ramón y Cajal, Juan de la Cierva, Beatriu de Pinós, ICREA. \\
\hline Estancias de investigación & \\
\hline Infraestructuras & $\begin{array}{l}\text { Premios de investigación, ayudas para asistencia a congresos, } \\
\text { acciones integradas, acciones complementarias. }\end{array}$ \\
\hline Otras ayudas
\end{tabular}


Asimismo, al estar toda la información correctamente clasificada, todas las salidas u outputs resultan mucho más ágiles y sencillas de elaborar. Un gestor de la información sabe las veces en un año que necesita listar, por ejemplo, los investigadores Ramón y Cajal que hay en la universidad. Con el sistema de TAIR sólo hay que introducir el número de la convocatoria en una consulta a la base de datos y se obtendrá rápidamente la información. Los balances de resultados que se elaboran anualmente en las unidades de gestión de la investigación también se pueden realizar de manera mucho más eficaz, puesto que los capítulos de investigación (Proyectos, Becas, Convenios, etc.), van implícitos en la clasificación que ofrece TAIR.

\section{Objetivos a corto $y$ medio plazo}

El objetivo más inmediato en el desarrollo de TAIR es reforzar la conexión con otras aplicaciones del ámbito de la investigación de la universidad. Actualmente los datos curriculares que se crean con TAIR ya se recogen en la aplicación de gestión curricular de la universidad, EINA. De esta manera se evita a los investigadores tener que introducir parte de sus actividades científicas.

Asimismo, TAIR tiene también conexión con SIGMA, el software que gestiona los alumnos de la universidad. Esta connexión entre aplicaciones es necesaria en cuanto a la selección de alumnos para la oferta de becas propias. TAIR coge los alumnos directamente de SIGMA, facilitando todos los datos que van a formar parte del expediente de la solicitud, tales como notas académicas, datos personales, director de tesis, etc.

Finalmente TAIR conecta también con la aplicación contable de la universidad, SUMMA, mediante un proceso diario y nocturno para traspasar los datos económicos de las ayudas concedidas, siendo la aplicación contable la que gestionará los movimientos económicos que se den durante la vida del proyecto.

La integración de TAIR con las aplicaciones de UAB aún no es plena. Se hace necesario, fundamentalmente, la integración con la aplicación que gestiona el personal investigador, HOMINIS. El movimiento de investigadores en una institución de un tamaño considerable como la UAB hace necesario un esfuerzo extraordinario para mantener al día el personal investigador en dos aplicaciones a la vez. Dicho esfuerzo supone asimismo destinar recursos de la universidad a duplicar datos que ya se encuentran en otra base de datos. Por tanto, el objetivo más inmediato es la integración de las aplicaciones de investigación y personal.

\section{Conclusiones: saliendo al mundo exterior}

En el ámbito de la gestión universitaria de la investigación hay unos actores que interactúan cíclicamente con las universidades. Nos referimos a los organismos estatales y autonómicos que financian la investigación. 
Con el desarrollo de las nuevas tecnologías es cada vez más habitual que estos organismos demanden a los investigadores que introduzcan directamente en sus portales las solicitudes de sus convocatorias. Esta novedad supone un nuevo marco para las universidades, porque quedan descapitalizadas de información. Es decir, no saben quién ni qué están solicitando sus investigadores, puesto que estos van directamente a las entidades financiadoras a demandar las ayudas, cuando tradicionalmente lo han hecho a través de las unidades de gestión de recursos de la investigación de sus instituciones. Es a posteriori cuando la universidad solicita a los organismos financiadores la información y la reintroduce en sus sistemas.

Esta nueva situación, como no es difícil de suponer, implica un menoscabo de fuerza de trabajo y tiempo para todos los implicados; investigadores, organismos financiadores y universidades. Al no estar perfectamente coordinados, estos actores a menudo efectúan el mismo trabajo en paralelo o bien tratan la misma información pero de manera dispersa y redundante.

En este sentido TAIR les va a facilitar la identificación de todas sus convocatorias y solicitantes, la confección de sus balances y sus estadísticas de éxito. Ello va a significar un gran avance en la gestión de la investigación pues actualmente estas actividades se llevan a cabo con herramientas caseras o bien con herramientas no pensadas para la gestión de la investigación sino para la gestión económica.

Pero no sólo eso. El escenario óptimo, que ya empieza a dibujarse con algunos proyectos como el Curriculum Vitae Normalizado de FECYT (Báez y otros 2008), será aquel en el que los datos se vuelquen en procesos automáticos de unas instituciones a otras, mediante protocolos de traspaso de datos, sin que las instituciones implicadas tengan que llevar a cabo un esfuerzo extra para recolectar su propia información o exportarla a instituciones foráneas.

Asimismo, el desarrollo de los certificados y firmas digitales por parte de administraciones y empresas incidirá en estos procesos de acumulación de datos electrónicos, dándoles una nueva dimensión. No en vano ya hace algún tiempo que empezamos a oír hablar de las tecnologías web 3.0 en las que la circulación de datos entre sistemas va a tener un papel primordial (López, 2009). Pensamos que un programa como TAIR va a facilitar los cambios que se avecinan en la gestión de los recursos de la investigación y a la sociedad en general.

\section{Notas técnicas}

El producto TAIR se ha desarrollado en lenguaje Java, siguiendo la especificación J2EE de SUN en la versión 6 y el SDK 1.6.

Se utiliza el paradigma conocido como Modelo Vista Controlador (MVC), mediante un modelo de tres capas, la ya mencionada J2EE, el servidor de aplicaciones SUN Glassfish y una tercera capa de acceso a datos, partiendo de la base de datos original (Ingres) y en desarrollo (Oracle). 
El desarrollo del producto ha ido a cargo de la empresa SIGMA, AIE.

EINA es la aplicación de gestión curricular de la UAB. Es una aplicación web desarrollada en java por la empresa IECISA, pero mantenida actualmente por SIGMA, AIE.

SIGMA es la aplicación de gestión académica de la UAB. Es una aplicación web desarrollada en java por la empresa Ibermática, pero mantenida actualmente por SIGMA, AIE.

HOMINIS es la aplicación de gestión de recursos humanos de la UAB. Es una aplicación web desarrollada en java por la empresa OCU.

SUMMA es la aplicación de gestión de los recursos económicos y de contabilidad de la UAB. Está desarrollada en un entorno SAP por la empresa IECISA.

\section{Bibliografía}

Álvarez Valladares, R. (2009): Informe Anual 2009. Red UGI. Madrid: CRUE.

Argimabu Vivó, Ll. (2004): El procesamiento documental de la información sobre investigación en Cataluña [en línea]. Hipertext.net, (2), [consultado el 15/02/2012].

Argimbau Vivó, Ll. (2006): Estudi de la informació especialitzada en recerca, desenvolupament i innovació $(\mathrm{R}+\mathrm{D}+\mathrm{I})$ a Catalunya [en línia]. Tesina DEA de la UAB. $<$ http:// www.recercat.net/bitstream/2072/3849/1/PROJECTE_DEF.pdf>. [Consultado el 15/05/2012].

Báez, J. M.; Peset, F.; Núñez, F., y Ferrer, A. (2008): CVN: normalización de los currículos científicos. El Profesional de la Información, vol. 17 (2), 213-220.

Baras Escolà, C. (2004): La gestión de la investigación desde el punto de vista de los gestores. XXII Jornadas de Gerencia Universitaria. Universidad de León, Octubre 2004. [en línea]. http://www.unileon.es/info_gral/congresos/gerencia/ponencias/dia21_4_1. pdf [consultado el 15/02/2012].

Iribarren-Maestro, I., y Sanz, E. (2010): Citation journal impact factor as a measure of research quality. En: Larsen, B. (ed.). The Janus Faced Scholar. A Festschrift in Honour of Peter Ingwersen. Special volume of the e-zine of the ISSI. Royal School of Library and Information Science, Copenhagen.

López, A. (2009): Digital Repositories Roadmap Review. IX Workshop Rebiun sobre Proyectos Digitales. Salamanca, España: Univ. de Salamanca.

Moros, A., y Bordons, M. (2003): La Memoria de Actividad como fuente de información bibliométrica en el estudio de una escuela politécnica superior. Revista Española de Documentación Científica, vol. 26 (2), 143-161. 